\title{
Advanced nursing practice in COPD exacerbations: the solution for a gap in Switzerland?
}

\author{
Gabriela Schmid-Mohler (10) ${ }^{1,2,3}$, Christian Clarenbach (10 ${ }^{2,3}$, Gabi Brenner ${ }^{4}$, \\ Malcolm Kohler ${ }^{2,3}$, Eva Horvath ${ }^{3}$, Marc Spielmanns $s^{5,6}$ and Heidi Petry ${ }^{1}$
}

Affiliations: ${ }^{1}$ Centre of Clinical Nursing Science, University Hospital Zürich, Zürich, Switzerland. ${ }^{2}$ Division of Pulmonology, University Hospital Zürich, Zürich, Switzerland. ${ }^{3}$ Medical Dept Heart-Vascular-Thorax, University Hospital Zürich, Zürich, Switzerland. ${ }^{4}$ Directorate of Nursing and Allied Health Professionals, University Hospital Zürich, Zürich, Switzerland. ${ }^{5}$ Pulmonary Medicine, Zürcher RehaZentren Klinik Wald, Zürich, Switzerland. ${ }^{6}$ Dept of Pulmonary Medicine, Faculty of Health, University Witten-Herdecke, Witten, Germany.

Correspondence: Gabriela Schmid-Mohler, Centre of Clinical Nursing Science and Division of Pulmonology, University Hospital Zürich, Ramistrasse 100, CH 8091 Zürich, Switzerland. E-mail: gabriela.schmiddusz.ch

\section{ABSTRACT}

Aim: This study aimed to address the need for adaptation of the current model of chronic obstructive pulmonary disease (COPD) care in Switzerland, particularly in regard to acute exacerbations, and how far an integrated approach involving advanced nursing practice can meet those needs.

Methods: A state analysis guided by the PEPPA framework was initiated by the Pulmonology Clinic of University Hospital Zürich. Literature describing the current provision of COPD care regarding exacerbations in Switzerland and international qualitative studies describing the patient perspective were systematically searched and summarised. The health providers' perspective was investigated in three focusgroup interviews.

Results: A lack of systematic and state-of-the-art support for patient self-management in Switzerland was described in literature and confirmed by the health providers interviewed. While care was assessed as being comprehensive and of good quality in each individual sector, such as inpatient, outpatient, rehabilitation and home settings, it was identified as being highly fragmented across sectors. The interview participants described day-to-day examples in which a lack of support in COPD self-management and fragmentation of care negatively affected the patients' disease management.

Conclusion: The necessity of coordinating the transition between healthcare sectors and self-management support and that these organisational boundaries should be addressed by a multi-professional team were identified. Initial evaluation indicates that advanced practice nurses potentially have the skill set to coordinate the team and address patients' self-management needs in complex patient situations. However, the legal foundation and a reimbursement system to ensure long-term implementation is not yet available.

@ERSpublications

Advanced practice nurses have the skills to meet needs in the treatment of \#AECOPD, such as transition between healthcare sectors and systematic self-management support, but the legal and financial framework is not present in Switzerland yet https://bit.ly/2wQysHV

Cite this article as: Schmid-Mohler G, Clarenbach C, Brenner G, et al. Advanced nursing practice in COPD exacerbations: the solution for a gap in Switzerland? ERJ Open Res 2020; 6: 00354-2019 [https://doi.org/10.1183/23120541.00354-2019]. 


\section{Introduction}

Chronic obstructive pulmonary disease (COPD) is one of the leading causes of death worldwide and has grown in importance over recent years [1]. COPD generally occurs alongside other concomitant diseases, including cardiovascular, musculoskeletal, metabolic and psychological comorbidities [2], requiring coordination of care. The onset of some comorbidities (e.g. cardiovascular) and exacerbations are directly influenced by patients' health behaviour, meaning that coordination and self-management support are two key areas in chronic disease management of COPD. To address those areas, new models of care have been implemented and evaluated in various countries, some of them using a nurse-led approach [3]. As this may also be a promising care model for the Swiss health system, careful evaluation is needed in this area.

\section{Economic burden of COPD in Switzerland}

400000 people with COPD live in Switzerland and COPD ranks as fourth on the list of leading causes for mortality [4]. In a Swiss cohort study with more than 300 patients, the average number of exacerbations per patient per year was 0.8 (SD 1.1), based on unpublished, post hoc analysis of SIEVI et al. [5]. Chronic care delivery accounts for $80 \%$ of costs in Switzerland and direct cost per COPD patient is estimated to be between CHF 1506 and CHF 5752 per patient per year, plus indirect costs due to absenteeism at work due to COPD between CHF 798 and CHF 5167 per patient per year [6]. Costs for stationary care (e.g. treatment of severe COPD exacerbations), range between CHF 485 and CHF 2986 per patient per year, which accounts for approximately half of the direct costs for COPD care [6].

\section{Effect of patients' behaviour on economic burden and COPD outcomes}

A substantial portion of costs and outcomes in COPD are directly influenced by patients' health behaviour. The number of acute exacerbations of COPD (AECOPDs) is decreased by influenza and pneumococcal vaccination compliance, higher adherence to inhalation, smoking cessation [7], and physical activity [8]. Mortality rates can be reduced by home oxygen therapy in selected cases [7], increasing physical activity [9] and physical capacity by following a pulmonary rehabilitation program [10]. The number of hospitalisations is reduced by patients' ability to recognise exacerbation symptoms and manage them by following an action plan [11]. Thus, the promotion of health behaviour plays a key role in the disease management of COPD.

\section{The role of advanced nursing practice}

Fostering healthy behaviour is one area of expertise of nurses, particularly advanced practice nurses (APNs) who provide patient coaching and guidance regarding patients' day-to-day self-management [12]. The International Council of Nurses defines a nurse practitioner/APN as 'a registered nurse who has acquired the expert knowledge base, complex decision-making skills and clinical competencies for expanded practice, the characteristics of which are shaped by the context and/or country in which s/he is credentialed to practice'. A Master's degree is recommended for entry level by the International Council of Nurses [13]. Expanded practice commonly refers to one area of expertise, pertaining either to a phenomenon (e.g. delirium) or a patient group (e.g. COPD), and may involve specialised assessments, performance of selected diagnostic interventions, ordering laboratory or diagnostic tests, prescription of medication under delegation or supervision of a physician, referral to specialised treatment, and/or coordination of services in complex situations [14].

\section{Systematic approach for APN role development}

Although the positive impact of APN services on patients' health and reduction of healthcare utilisation has been shown in international research, the overall body of evidence is not robust and its effect on patient outcomes is poorly understood. Therefore, a systematic approach, namely a participatory, evidence-based, patient-centred process for the development, introduction and evaluation of APN roles (PEPPA) has been highlighted [15, 16]. Following a systematic framework in the development of an APN role fosters critical evaluation as to whether other models of care may be more suitable, functional or cost-effective for patient care. The PEPPA framework describes nine steps from 1) defining the patient population and describing the current model of care; 2) identifying stakeholders and patient perspectives; 3 ) describing the need for a new model of care; 4) identifying problems and goals to improve the model of care; 5) defining the new model of care and the APN role within this model; 6) planning implementation strategies; 7) implementing the APN role; 8) evaluation; and 9) long-term monitoring of the role.

The aim of this study was to describe the deficits and the need for adaptation of the current model of COPD care in Switzerland, particularly in regard to AECOPDs. This will serve as a basis for critically evaluating the potential of an APN approach to meet those needs. 


\section{Methods and materials Study design}

To address the first aim, we followed the first four (of nine total) steps of the PEPPA framework and adapted them slightly (table 1). The PEPPA framework provides little guidance regarding the research method. For this project, we selected the methods for each step that suited our predefined time frame (6 months) and available resources (maximum two working days per week during those 6 months), and which promised to provide a robust basis for the new model of care.

We used a combination of methods. In the first step, we performed two literature reviews. On the one hand, we reviewed existing quantitative evidence that described COPD care in Switzerland and on the other hand, we synthesised qualitative studies that described patients' experience in COPD. In the second step, we investigated health professionals' perspectives using focus-group interviews. During the interviews, we presented the summarised evidence from step 1 to the health professionals and assessed their perspective on how the identified needs could be addressed in the Swiss setting.

In a final step, all three data sources were synthesised, which provided the basis for the development of the new model of care (figure 1).

\section{Scoping review of quantitative studies describing COPD care in Switzerland}

The aim of the review was to identify quantitative studies describing the current service provision for patients experiencing an exacerbation of COPD in Switzerland, including adherence to guidelines (e.g. guidelines from the Global Initiative for Chronic Obstructive Lung Disease). For this purpose, we performed a scoping review of the evidence published in the previous 10 years. Studies were identified by using the search terms (("Switzerland"[Mesh]) AND "pulmonary disease, chronic obstructive"[Mesh]) AND (“disease progression"[Mesh] OR COPD exacerbation) in MEDLINE/PUBMED in February 2018, repeated on August 16, 2019.

Of the identified 33 studies, only one suited our aim completely [17]. Two further studies investigated adherence to guidelines that were not specific regarding exacerbations; however, both were published before $2009(n=1)$ [18], or reviewed evidence before $2009(n=1)$ [19] and were therefore not included in the review. The other studies did not cover the topic of interest $(n=30)$. Quality of the identified study was assessed using the Critical Appraisal Skills Programme checklist [20].

\section{Patient perspective: narrative review of qualitative studies}

A narrative review with the aim of describing patients' experience of an exacerbation was performed. Search terms were defined using the PICO format (population: COPD; phenomena of interest: patient experience; context: during acute exacerbations, at home or in hospital). On February 20, 2018, the databases MEDLINE/PUBMED, CINAHL, EMBASE/OVID SP, PSYCINFO and ASSIA were searched with the following search terms: (interview ${ }^{\star}$ OR experience ${ }^{\star}$ OR qualitative) AND pulmonary disease, chronic

TABLE 1 Methods used to assess the need for an advanced practice nurse following steps 1-4 of the PEPPA framework

\begin{tabular}{|c|c|c|c|}
\hline Step & PEPPA framework [15] & Adapted approach in our study & Method \\
\hline 1 & $\begin{array}{l}\text { Define patient population } \\
\text { Describe current model } \\
\text { of care }\end{array}$ & $\begin{array}{l}\text { Define patient population } \\
\text { Describe current model of care and } \\
\text { determine need for a new model of } \\
\text { care (based on evidence) }\end{array}$ & $\begin{array}{l}\text { Scoping review of quantitative } \\
\text { studies that describe COPD } \\
\text { care in Switzerland }\end{array}$ \\
\hline 2 & $\begin{array}{l}\text { Identify stakeholders } \\
\text { Recruit patients }\end{array}$ & $\begin{array}{l}\text { Identify patient perspective on current } \\
\text { model of care and determine need } \\
\text { for new model of care }\end{array}$ & $\begin{array}{l}\text { Synthesis of qualitative } \\
\text { studies of patient } \\
\text { perspectives (from various } \\
\text { countries) }\end{array}$ \\
\hline 3 & $\begin{array}{l}\text { Determine need for a new } \\
\text { model of care } \\
\text { Identify priority problems } \\
\text { and goals to improve } \\
\text { the model of care }\end{array}$ & $\begin{array}{l}\text { Identify stakeholders perspective on } \\
\text { current model of care and their } \\
\text { perceived view of the need for new } \\
\text { model of care and harmonise with } \\
\text { identified perspectives from } \\
\text { evidence and patient perspective }\end{array}$ & $\begin{array}{l}\text { Focus-group interviews with } \\
\text { stakeholders }\end{array}$ \\
\hline
\end{tabular}

PEPPA: participatory, evidence-based, patient-centred process for the development, introduction and evaluation of advanced practice nurse roles. 


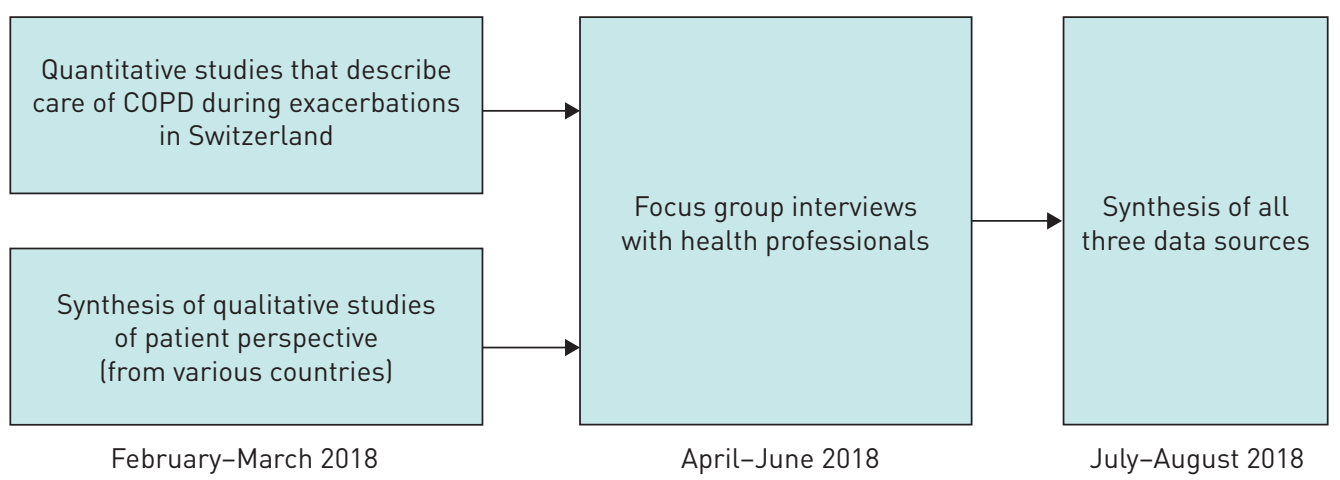

FIGURE 1 Overview and timeline of different methods.

obstructive AND exacerbation*. Studies that were included explored the patient experience in connection with an AECOPD and were published in a peer-reviewed journal. Studies were excluded if they referred to a specific intervention, were written in a language other than German or English, included perspectives other than those of patients, were solely congress abstracts and had been published in 2011 or earlier. The restriction of time was chosen because a synthesis of qualitative research summarising the earlier studies was published in 2012 [21]. Of 1331 screened articles, 15 qualitative studies fulfilled the criteria and were included in the review. As the quality of the study was not an inclusion or exclusion criteria, no quality appraisal was performed. Data were analysed with a content analytical procedure. The first level of the index for the analysis and the synthesis of the data sources was developed deductively from the research aims and the Chronic Care Model [22] and had the following four categories: 1) influencing factors for the model of care; 2) organisation of care; 3) managing transitions; and 4) self-management support of patients and caregivers. Subcategories (on level 2) were then inductively derived from the data. Data in the same category were summarised.

\section{Health providers' perspective \\ Sample/participants}

Three focus interviews with 6-10 health professionals each were planned. Purposive sampling was applied with a broad range from different health professions as criteria.

\section{Data collection}

Health professionals were invited by e-mail to the focus-group interviews. Participants were informed of the purpose and procedure of the interviews, that participation was voluntary, and anonymity was guaranteed. The three interviews were scheduled between April and June 2018; two at the University Hospital Zürich and one at a rehabilitation centre in the area. The interview guide was 1) Where do you see deficits and needs in the current care of COPD patients as regards prevention and management of AECOPDs? and 2) From your perspective, what adaptations should occur in COPD care in future? In the second hour of the interview, participants received two thematic prompts, which were results from a study describing the performance of evidence-based COPD care bundles in the Swiss setting [17] and the review of the patients' experience of a COPD exacerbation.

\section{Ethical considerations}

Data collection involving health professionals does not fall under Human Research Law in Switzerland, therefore no submission to an ethics commission was undertaken. The audio records were transferred directly after the interview onto a password-protected computer within the University Hospital Zürich. The interviews were transcribed, with the transcripts being coded and pseudonyms being used in the transcript. All person- and setting-related information was anonymised.

\section{Data analysis}

Data were analysed with a content analytical procedure following the same index as for patients' perspective. Data in the same category were summarised. 


\section{Results}

The current model of care and its deficits

Scoping review of quantitative studies describing COPD care in Switzerland

The identified study investigated the degree of implementation of acute and post-acute interventions for AECOPDs. While diagnostic and therapeutic interventions were well and broadly realised, interventions to promote health behaviour were only implemented to a minimal extent in the hospital setting [17]. Only $1.9 \%$ of patients received some form of self-management support, and reassessment of inhaler technique took place in only $19.8 \%$ of patients. This striking lack of self-management support has also been reported in international literature [23].

In addition to the systematic scoping review, we identified two studies that described the adaptation, implementation and evaluation of the Canadian-developed 6-week self-management programme Living Well with COPD in several cantons. The adapted programme showed a consistent positive effect on quality of life and to some extent also on the prevention of new exacerbations [24, 25].

Synthesis of qualitative studies describing the patients' perspective

Characteristics

Fifteen qualitative studies met the criteria and were included in the narrative review. Publication dates were between 2012 and 2017 and were performed in the United Kingdom (4), Norway (3), USA (3), Denmark (2), the Netherlands (1), Australia (1) and Taiwan (1) and included a total of 326 patients with COPD Global Initiative for Chronic Obstructive Lung Disease stage 1-4 (140 female and 186 male).

\section{Handling burdensome emotions}

Fear was reported as the greatest source of distress for COPD patients. The knowledge that COPD is a progressive disease troubled many patients. They were concerned about suffering in future, death, loss of independence and missing important events in their lives [26, 27]. Patients reported exacerbations of COPD as especially frightening episodes during which anxiety increases significantly and could result in panic and mortal fear [26, 28-30]. Repeated experience of an exacerbation led over the years to a steady increase in fear of breathlessness and death [31]. The fear negatively affected the patient's ability to apply self-management strategies to reduce symptom distress [29]. Additionally, patients reported feelings of guilt and depression [32]. In handling their burdensome emotions, they experienced the following as helpful: trust in their health care team, feeling heard and respected by their health care team, being involved in decision-making, family support, faith in God, steady advances in medical science and organising their affairs in case of death [27].

\section{Managing COPD symptoms and therapy in daily life}

Some patients reported putting great effort in following the recommendations of health professionals and experienced an ongoing battle to maintain their state of health and well-being [26]. While the lack of energy aggravates daily self-management [33], practical and emotional support from others was helpful [34]. Patients struggled in particular with the recommendation of smoking cessation [35] and experienced health professionals as being strongly focused on this topic [30]. Smokers acknowledged smoking as contributing to COPD, but felt frustrated and/or deceived because smoking cessation would not stop the progression of COPD [27] or because they experienced relapses [33]. However, some patients did report motivation to change smoking behaviour after having experienced a pulmonary exacerbation [30]. Generally, patients experiencing dyspnoea put considerable effort into managing it and employed several strategies such as the use of inhalers, nebulisers, continuous positive airway pressure or bilevel positive airway pressure machines, and breathing techniques [36], as well as mental strategies for managing fear and panic, which they had acquired in courses or through their own experiences [28, 30]. Despite these efforts, patients experienced dyspnoea as difficult to control, which led to frustration and restriction of daily physical activity [35]. Patients who had home oxygen therapy in particular experienced the transportation of the devices as burdensome, which limited their participation in activities [34, 35], also leading to restriction in social relationships [27]. Due to the disease and their symptoms (e.g. dyspnoea), participants could not engage in their hobbies or the activities they liked to do and consequently felt trapped in their homes and lonely $[26,27,32,34]$. Younger patients experienced a significant reduction in their performance capacity in connection with an exacerbation, so that they were unable to fulfil their tasks at work or within the family, which alarmed them [32].

Recognising a pulmonary exacerbation and acting accordingly

Recognising an exacerbation and differentiating it from the day-to-day variation of symptoms was perceived as difficult $[29,36,37]$, with the evaluation of the exacerbation's severity being experienced as especially challenging. The evaluation was influenced by the patients' varying attitudes and beliefs as to 
which symptoms and signs indicated a severe condition, which in turn affected the time point at which they contacted their health care team $[33,38]$. Other reasons for not contacting their team was a wish to manage the situation on their own, avoiding a hospitalisation, feeling ashamed because of continued smoking, other priorities in life and not wanting to disturb health professionals [29, 31, 33]. Help-seeking behaviours were further impacted by earlier experiences of an exacerbation and the patients' trust in the health care team [33]. Of those patients who had received an action plan that describes AECOPD symptoms and strategies on how to manage an AECOPD, the vast majority could not remember when they had received the action plan and how they were instructed to use it. Nevertheless, most patients seem to have understood that they should take medication on their own when getting worse [38]. Some patients had an ambivalent attitude toward taking cortisone or antibiotics because of the side effects. Patients were reluctant to contact health professionals or to take medication as prescribed although being aware of the importance of the treatment $[29,37,38]$. Despite doubts regarding medication, patients perceived the possibility of initiating medication on their own as generally positive because they did not have to arrange an urgent care visit and could avoid travelling to their physician, which gave them a sense of security [38].

\section{Experience of hospitalisations and pulmonary rehabilitation}

Patients experienced hospitalisations as distressing and uncontrollable events [33]. Various patients reported that they experienced a reduced mental or even delirious state during an exacerbation [30, 39]. They reported an intense need for a sense of security and experienced being or left alone in the room as difficult [30]. As soon as their condition improved, a need for information regarding their condition and treatment options arose [33] with patients wishing to be better informed by health professionals [21]. If health professionals suggested participating in a pulmonary rehabilitation programme, patients evaluated the recommendation in the context of their personal living situation, their previous experiences with physiotherapy and narratives from other individuals [32]. Patients' reasons for declining rehabilitation were having the impression that the physical activity could aggravate dyspnoea, that they had already integrated enough activity into their daily life, a perception of not being really ill, having no wish to be confronted with COPD, or difficulties in combining it with work [40]. After an exacerbation, patients needed several weeks for recovery to reach their previous state [31].

\section{TABLE 2 Characteristics of health professionals}

\begin{tabular}{|c|c|c|c|}
\hline & Interview 1 & Interview 2 & Interview 3 \\
\hline Length of interview min & 80 & 80 & 80 \\
\hline Location & Hospital & Hospital & $\begin{array}{l}\text { Rehabilitation } \\
\text { centre }\end{array}$ \\
\hline Total number of participants & 6 & 6 & 6 \\
\hline Female/male & $6 / 0$ & $2 / 4$ & $4 / 2$ \\
\hline \multicolumn{4}{|l|}{ Age years } \\
\hline $30-40$ & 3 & 1 & 2 \\
\hline $40-50$ & 0 & 2 & 1 \\
\hline $50-60$ & 3 & 3 & 2 \\
\hline$>60$ & 0 & 0 & 1 \\
\hline $\begin{array}{l}\text { Years of expertise with patients with COPD } \\
\text { range (median) }\end{array}$ & $7-20(11)$ & $4-25(10)$ & $3-26(8)$ \\
\hline \multicolumn{4}{|l|}{ Profession } \\
\hline Pulmonology nurse & 2 & 1 & 2 \\
\hline CNS palliative care & 0 & 1 & 0 \\
\hline Pulmonologists & 2 & 1 & 1 \\
\hline Physiotherapist & 0 & 1 & 1 \\
\hline Nutritionist & 0 & 0 & 1 \\
\hline Social worker & 0 & 0 & 1 \\
\hline Pastoral care & 1 & 1 & 0 \\
\hline Nursing home care & 0 & 1 & 0 \\
\hline Pulmonary League nurse $\mathrm{e}^{\#}$ & 1 & 0 & 0 \\
\hline \multicolumn{4}{|c|}{$\begin{array}{l}\text { Data are presented as } n \text {, unless otherwise stated. CNS: clinical nurse specialist. \#: The Pulmonary League } \\
\text { is a nonprofit organisation that represents a centre of competence for respiratory diseases in Switzerland. } \\
\text { It coordinates preventive policies (actions) and offers a variety of therapeutic services, mostly delivered } \\
\text { by nurses. }\end{array}$} \\
\hline
\end{tabular}




\section{Health professionals \\ Characteristics}

The views of 18 individuals working in different clinical settings (inpatient, outpatient, rehabilitation and home care) were investigated in three focus-group interviews. Sample characteristics are displayed in table 2.

\section{Current model of care}

Overall, the 18 participants rated the care in the individual sectors (inpatient, outpatient, rehabilitation, home care) as comprehensive and of good to very good quality. However, all evaluated the care across the sectors as highly fragmented. This is aggravated by the fact that due to the high prevalence of COPD patients, a large number of health care providers are involved in the treatment of COPD patients, especially in primary care. Further, this is a population suffering from multiple comorbidities often being treated by various specialists. Participants observed that patients treated as outpatients or on a nonpulmonary ward often do not receive treatment that adequately addresses COPD-specific care needs. Health professionals felt that detailed assessments and care planning were performed in each sector but not followed by the next sector. They perceived this as a waste of resources. They observed that some patients arrived with nonrealistic expectations (e.g. pulmonary rehabilitation) and hypothesised that this is probably caused by insufficient information of the patients by the previous caregiver (e.g. hospital ward).

The participants of the focus groups described a lack of systematic, coordinated and state-of-the-art support for patients' self-management. They perceived a crucial need for supporting patients in acquiring knowledge and skills regarding their disease, but acknowledged that this kind of support is time intensive and rarely available in daily practice. Some felt that patients are confronted with many different and uncoordinated expectations and recommendations from health professionals with the lack of coordination regarding the recommendations (e.g. smoking cessation) leading to resistance on the part of the patient. Some health professionals also critically indicated that they did not feel sufficiently prepared for supporting behaviour change in patients and that patients are not always open to education and counselling. Exacerbation action plans were seen as an important element, but they were not well implemented and those few patients who had one did not seem to follow them in decision-making. The participants described examples from everyday practice in which a lack of support for COPD self-management and the fragmentation of care negatively affected the patients' disease management. The incorrect use of inhalation devices and inappropriate nutrition recommendations were mentioned as examples, resulting in both cases in adverse outcomes for patients. They further highlighted that patients with COPD suffer from emotional distress, which may be aggravated by social isolation and feelings of guilt and fear from threatening symptoms such as dyspnoea. They reported that the screening of emotional distress in the current model of care is not systematically implemented and if psychological support is offered to patients, they often decline it. Current rehabilitation programmes in Switzerland focus mainly on physical activity and rarely take day-to-day management of COPD into account.

\section{The future model of care}

After having presented the main results of the previous two literature reviews, the health professionals identified three key areas that should be addressed in the future model of care. These are described in the following sections.

\section{Coordination of care}

There was complete consensus that the coordination and communication between the providers is the key area for improvement and must be addressed in a future model of care. The health professionals emphasised that "We have to develop a culture that focuses beyond the doorstep of each institution." Health professionals assessed the potential for improving patient outcomes as high in this area, but acknowledged that this sort of coordination will be time intensive and that it needs the legitimation of the care team. The coordinating person should also provide and ensure the access to COPD-specific care in the primary care setting, including the outpatient care and home settings, but also on wards that are not specialised for pulmonary medicine. The interviewees saw two possibilities: either the coordinating person provides the COPD-specific care by contacting the patient directly or educates and trains the health professional on site.

\section{State-of-the-art support of self-management}

There was further consensus that patients need self-management support that is ongoing and more holistic. They critically assessed that especially outpatient rehabilitation programmes should focus not only on physical activity, but also include a range of relevant topics, including inhalation, nutrition and management of burdensome emotions as recommended by the Swiss Pulmonary Medicine Association [41]. However, the health professionals highlighted that pulmonary rehabilitation is just one pillar of 
self-management support, but that ongoing support at home is necessary in order to enable patients to transfer knowledge and behaviour change in their daily routine. Especially patients with home oxygen need ongoing support so that they integrate the therapy into their daily lives without detrimental effects on social relationships. The interviewees emphasised again that addressing self-management topics should be coordinated by one person and that the person must have excellent communication skills and be knowledgeable about how to foster behaviour change.

\section{Screening and management of psychiatric comorbidities}

The interviewees agreed that a systematic screening of emotional burden should be established, and psychological support offered. They suggested a screening of emotional distress by a health professional who is known to the patient and has ideally established a trustful relationship. They assume that this will enhance the probability that patients would accept psychological support.

Regarding the structure of the new model of care, the interviewees suggested that one person should be assigned to each patient to coordinate with all of the other health professionals, plan self-management support systematically and address new topics (such as palliative care services or advance directives) over the course of the disease. The health professionals assessed that the coordination person must have a broad skill set, including medical knowledge about COPD and important comorbidities, decision-making skills and good communication skills. They suggested that the coordinating person should be a physician or an ANP. Due to the high number of patients, a team approach involving several persons will be necessary. The health professionals suggested that each patient should be seen at least once per year in stable phases and that the frequency of consultation shall be adapted in instable phases. Ideally, the coordinating service should be aligned to one specific institution, but must have the legitimation to access the different sectors.

\section{Discussion}

This study describes the current deficits and needs in COPD care in Switzerland based on two literature searches and actual experiences of health professionals. Three key areas were identified that should be addressed in the future model of care, which were coordination of COPD-specific care, state-of-the-art self-management support and screening and management of emotional burden.

Interventions to promote health behaviour (e.g. smoking cessation, after AECOPDs) are not only poorly implemented in Switzerland, but also in various other countries [23, 42]. The interviewed Swiss health professionals reported poor confidence in their skills to promote behaviour change as a main barrier. Reviewing international evidence confirmed that this lack of skills is an area for improvement [43]. The promotion of behaviour change needs complex skills and patients highlighted the importance of a trustful und respectful relationship to health professionals and mentioned openness to behaviour change after exacerbation. A triangulation of these three perspectives (scientific literature, patients' and health professionals' perspective) indicates that a coordinated approach for self-management is the key to success, especially longer term.

Across various countries, coordinated and self-management-focused models have been shown to be effective in improving clinical results: In a cluster randomised study, which included 22 hospitals in three countries, the implementation of a care pathway reduced the short-term readmission rate in the 11 intervention hospitals. In contrast to the control group hospitals, the care pathway of the 11 hospitals of the intervention group included significantly more interventions supporting smoking cessation, education regarding inhalation, nutrition assessments and interventions in patients with underweight, more follow-up appointments and, when needed, support at home [44]. Furthermore, a meta-analysis including 26 studies from 11 countries demonstrated that integrated disease management improved quality of life, exercise capacity and reduced the hospitalisation rate internationally [45]. The costs of integrated care with moderate to intensive support in self-management range between GBP 500 and GBP 600 per patient per exacerbation in the UK setting (including costs for physician and specialised nurse) [46], which seem to be low at first. No cost-benefit analysis for integrated care models for AECOPDs is available for Switzerland and is still needed.

Fear was reported as the most burdensome aspect of COPD from the patient perspective, which was confirmed by health professionals. They estimated that while anxiety and depression is a major topic in this population, it is often not recognised and is underdiagnosed. Elevated levels of depression and anxiety in COPD have been reported internationally, especially for those experiencing repeated AECOPDs [47]. As recommendations of the frequency and methods of screening are not provided in current COPD guidelines [7] they are scarcely addressed in current models of AECOPD care. Different approaches in screening and management of anxiety and depression have been reported across populations. For example, in patients with cystic fibrosis, an annual screening with the Patient Health Questionnaire and Generalised 
Anxiety Disorder 7 Questionnaire is recommended [48] and has been found feasible and well-accepted by patients, although assessed as time intensive by staff in clinical practice [49]. In cancer, the distress thermometer (a one-item screening tool) has been broadly implemented [50]. These examples for screening and management of emotional distress may function as a framework for the development of a concept for the new model of care for COPD patients.

On first evaluation, the skill set of an APN fits the identified needs for adaptation of a future model of care. However, if using an APN approach, the following points have to be considered. First, successful programs used a multi-professional approach [45], indicating the need for a close collaboration within the core team. A team that should include at least a physician, a physiotherapist and the APN. Depending on patient's needs, they can be supplemented with additional professionals, such as a nutritionist, social work, psychotherapist or pastoral care. Second, the costs for self-management support and coordination activities are not fully covered by health insurance (accounting system) in some countries, including Switzerland. Services delivered in own authorities by APNs are not regulated in legislation in Switzerland, probably one reason why no accounting system for APN services exists. The main reason for this omission may be the relatively short time since the introduction of the APN role in Switzerland (since 2000). Other Organisation for Economic Cooperation and Development members such as Ireland, the United Kingdom, the Netherlands, Canada, Australia, New Zealand and the United States, have a long-standing tradition of APN roles [51]. Currently, various efforts are in progress at this time to clarify the situation at the national and international level [51]. Not addressing this lack of clarity will be a critical barrier for the adoption and the long-term sustainability of an APN-led programme. Third, in various countries, including Switzerland, care models are traditionally physician-led. The acceptance of an APN-led care model may therefore pose a challenge for its adoption and should be addressed early in the development of the programme.

\section{Limitations}

Our methodological approach provided a solid foundation for the evaluation of an APN approach within the set timeframe. The main limitation is that we synthesised the perspective of patients and did not interview patients ourselves; therefore, the needs are described in general and are not specific to the Swiss setting.

\section{Conclusion}

Coordination of transition between acute, rehabilitative and home care, support of self-management and screening of emotional burden have been identified as the three key areas, all with high potential for improving patient outcomes and consequently lowering costs. Internationally, those programmes applying a multi-professional approach were the most promising for patient outcomes, in which an APN may function as a partner next to the patient and family and coordinate between different health professionals. This is a promising approach for the Swiss setting as well, especially as APNs are specialised in complex chronic care provision. However, legislative and accounting systems do not yet provide the necessary framework for such solutions and therefore the long-term sustainability of such programmes is in question.

Acknowledgements: We thank Jane White for proofreading assistance. We thank Andrea Tobler from the University of Applied Science St Gallen (St Gallen, Switzerland) for her contribution to reviewing and synthesising qualitative evidence. We thank the experts who participated in the focus-group interviews: Kathrin Signer from Lunge Zürich (Pulmonary League; Zürich, Switzerland); Rahel Jenkins from Spitex Zürich Limmat AG (Zürich, Switzerland); Stefanie Buerge and Ursula Stinglwagner from the Zürcher RehaZentren Klinik Wald (Zürich, Switzerland); and Monika Anderegg, Elisabeth Cohen, Markus Feuz, Ralf Schiemer, Andrea Schneider, Anne-Christin Stoewhas, Barbara Oberholzer and Claudia Weber from the University Hospital Zürich (Zürich, Switzerland).

Conflict of interest: G. Schmid-Mohler reports nonpersonal fees, paid to the University Hospital, for counselling from GSK, outside the submitted work; and a research grant from the Lunge Zürich for the investigator-initiated project NICCO (nurse-led integrated care COPD). C. Clarenbach reports personal fees from Roche, Novartis, Boehringer, GSK, AstraZeneca, Sanofi, Vifor and Mundipharma, outside the submitted work. G. Brenner has nothing to disclose. M. Kohler reports personal fees from Boehringer Ingelheim, Novartis, AstraZeneca, GSK, Roche, Bayer and Mundipharma, outside the submitted work; and a research grant from Lunge Zürich for the investigator-initiated project NICCO. E. Horvath has nothing to disclose. M. Spielmanns has nothing to disclose. H. Petry has nothing to disclose.

\section{References}

1 Global Burden of Disease Study Collaborators. Global, regional, and national age-sex specific mortality for 264 causes of death, 1980-2016: a systematic analysis for the Global Burden of Disease Study 2016. Lancet 2017; 390: 1151-1210.

2 Smith MC, Wrobel JP. Epidemiology and clinical impact of major comorbidities in patients with COPD. Int $J$ Chron Obstruct Pulmon Dis 2014; 9: 871-888. 
3 Baker E, Fatoye F. Clinical and cost effectiveness of nurse-led self-management interventions for patients with COPD in primary care: a systematic review. Int J Nurs Stud 2017; 71: 125-138.

4 Federal Office of Public Health (FOPH). Chronic Respiratory Diseases (German). www.bag.admin.ch/bag/de/ home/krankheiten/krankheiten-im-ueberblick/chronische-atemwegserkrankungen.html Date last accessed: July 27, 2019. Date last updated: 2018.

5 Sievi NA, Kohler M, Thurnheer R, et al. No impact of exacerbation frequency and severity on the physical activity decline in COPD: a long-term observation. Int J Chron Obstruct Pulmon Dis 2019; 14: 431-437.

6 Wieser S, Tomonaga Y, Riguzzi M, et al. The costs of non-communicable diseases in Switzerland: Final Report (German). Winterthur, Zurich University of Applied Science, 2014.

7 Global Initiative for Chronic Obstructive Lung Disease. Global strategy for the diagnosis, management, and prevention of chronic obstructive pulmonary disease: 2019 report.

8 Moy ML, Teylan M, Weston NA, et al. Daily step count predicts acute exacerbations in a US cohort with COPD. PLOS ONE 2013; 8: e60400.

9 Waschki B, Kirsten A, Holz O, et al. Physical activity is the strongest predictor of all-cause mortality in patients with COPD: a prospective cohort study. Chest 2011; 140: 331-342.

10 Puhan MA, Gimeno-Santos E, Cates CJ, et al. Pulmonary rehabilitation following exacerbations of chronic obstructive pulmonary disease. Cochrane Database Syst Rev 2016; 12: CD005305.

11 Lenferink A, Brusse-Keizer M, van der Valk PD, et al. Self-management interventions including action plans for exacerbations versus usual care in patients with chronic obstructive pulmonary disease. Cochrane Database Syst Rev 2017; 8: CD011682.

12 O'Grady ET, Johnson JE. Guidance and coaching. In: Tracy MF, O'Grady ET, eds. Hamric and Hanson's Advanced Practice Nursing: An Integrative Approach. 6th Edn. St. Louis, Elsevier Inc., 2019; pp. 179-202.

13 International Council of Nurses (ICN). Definition and Characteristics of the Role. 2019 Date last accessed: May 27, 2020. https://international.aanp.org/Practice/APNRoles.

14 Tracy MF. Direct clinical practice. In: Tracy MF, O'Grady ET, eds. Hamric and Hanson's Advanced Practice Nursing: An Integrative Approach. 6th Edn. St. Louis, Elsevier Inc., 2019; pp. 143-178.

15 Bryant-Lukosius D, Dicenso A. A framework for the introduction and evaluation of advanced practice nursing roles. J Adv Nurs 2004; 48: 530-540.

16 Bryant-Lukosius D, Spichiger E, Martin J, et al. Framework for evaluating the impact of advanced practice nursing roles. J Nurs Scholarsh 2016; 48: 201-209.

17 Markun S, Franzen DP, Dalla Lana K, et al. Acute exacerbated COPD: room for improvement in key elements of care. Int J Chron Obstruct Pulmon Dis 2017; 12: 2969-2975.

18 Fritsch K, Jacot ML, Klarer A, et al. Adherence to the Swiss guidelines for management of COPD: experience of a Swiss teaching hospital. Swiss Med Wkly 2005; 135: 116-121.

19 Steurer-Stey C, Dallalana K, Jungi M, et al. Management of chronic obstructive pulmonary disease in Swiss primary care: room for improvement. Qual Prim Care 2012; 20: 365-373.

20 Critical Appraisal Skills Programme. CASP Case Control Study Checklist. 2018. Date last accessed: May 27, 2020. https://casp-uk.net/wp-content/uploads/2018/01/CASP-Case-Control-Study-Checklist-2018.pdf.

21 Giacomini M, DeJean D, Simeonov D, et al. Experiences of living and dying with COPD: a systematic review and synthesis of the qualitative empirical literature. Ont Health Technol Assess Ser 2012; 12: 1-47.

22 Wagner EH, Austin BT, Von Korff M. Improving outcomes in chronic illness. Manag Care Q 1996; 4: 12-25.

23 Seys D, Bruyneel L, Decramer M, et al. An international study of adherence to guidelines for patients hospitalised with a COPD exacerbation. COPD 2017; 14: 156-163.

24 Steurer-Stey C, Dalla Lana K, Braun J, et al. Effects of the "Living well with COPD" intervention in primary care: a comparative study. Eur Respir J 2018; 51: 1701375.

25 Carron T, Bridevaux PO, Lorvall K, et al. Feasibility, acceptability and effectiveness of integrated care for COPD patients: a mixed methods evaluation of a pilot community-based programme. Swiss Med Wkly 2017; 147: w14567.

26 Walker S, Andrew S, Hodson M, et al. Stage 1 development of a patient-reported experience measure (PREM) for chronic obstructive pulmonary disease (COPD). NPJ Prim Care Respir Med 2017; 27: 47.

27 Schroedl CJ, Yount SE, Szmuilowicz E, et al. A qualitative study of unmet healthcare needs in chronic obstructive pulmonary disease. A potential role for specialist palliative care? Ann Am Thorac Soc 2014; 11: 1433-1438.

28 Halpin D, Hyland M, Blake S, et al. Understanding fear and anxiety in patients at the time of an exacerbation of chronic obstructive pulmonary disease: a qualitative study. JRSM Open 2015; 6: 2054270415614543.

29 Korpershoek Y, Vervoort S, Nijssen L, et al. Factors influencing exacerbation-related self-management in patients with COPD: a qualitative study. Int J Chron Obstruct Pulmon Dis 2016; 11: 2977-2990.

30 Torheim $H$, Kvangarsnes $M$. How do patients with exacerbated chronic obstructive pulmonary disease experience care in the intensive care unit? Scand J Caring Sci 2014; 28: 741-748.

31 Chin ED. The COPD exacerbation experience: a qualitative descriptive study. Appl Nurs Res 2017; 38: 38-44.

32 Harrison SL, Robertson N, Apps L, et al. 'We are not worthy' - understanding why patients decline pulmonary rehabilitation following an acute exacerbation of COPD. Disabil Rehabil 2015; 37: 750-756.

33 Andersen IC, Thomsen TG, Bruun P, et al. The experience of being a participant in one's own care at discharge and at home, following a severe acute exacerbation in chronic obstructive pulmonary disease: a longitudinal study. Int J Qual Stud Health Well-being 2017; 12: 1371994.

34 Thorpe O, Kumar S, Johnston K. Barriers to and enablers of physical activity in patients with COPD following a hospital admission: a qualitative study. Int J Chron Obstruct Pulmon Dis 2014; 9: 115-128.

35 Chang YY, Dai YT, Chien NH, et al. The lived experiences of people with chronic obstructive pulmonary disease: a phenomenological study. J Nurs Scholarsh 2016; 48: 466-471.

36 Brandt CL. Study of older adults' use of self-regulation for COPD self-management informs an evidence-based patient teaching plan. Rehabil Nurs 2013; 38: 11-23.

37 Williams V, Hardinge M, Ryan S, et al. Patients' experience of identifying and managing exacerbations in COPD: a qualitative study. NPJ Prim Care Respir Med 2014; 24: 14062.

38 Laue J, Melbye H, Risor MB. Self-treatment of acute exacerbations of chronic obstructive pulmonary disease requires more than symptom recognition - a qualitative study of COPD patients' perspectives on self-treatment. BMC Fam Pract 2017; 18: 8 . 
39 Kvangarsnes $\mathrm{M}$, Torheim $\mathrm{H}$, Hole $\mathrm{T}$, et al. Narratives of breathlessness in chronic obstructive pulmonary disease J Clin Nurs 2013; 22: 3062-3070.

40 Mathar H, Fastholm P, Lange P, et al. Why do patients decline participation in offered pulmonary rehabilitation? A qualitative study. Clin Rehabil 2017; 31: 1674-1683.

41 Swiss Society for Pulmonology. Accreditation Requirements for Pulmonary Rehabilitation Programs from the Swiss Society for Pulmonology (German). 2017 Date last accessed: May 27, 2020. www.pneumo.ch/files/pneumo/ pdf/fachpersonen/fortbildung/pulmonale_rehabilitation/Anforderung_Pulm_Rehabilitation_D_NEU.pdf.

42 Epstein D, Barak-Corren Y, Isenberg Y, et al. Clinical decision support system: a pragmatic tool to improve acute exacerbation of COPD discharge recommendations. COPD 2019; 16: 18-24.

43 Harrison SL, Goldstein R, Desveaux L, et al. Optimizing nonpharmacological management following an acute exacerbation of chronic obstructive pulmonary disease. Int J Chron Obstruct Pulmon Dis 2014; 9: 1197-1205.

44 Vanhaecht K, Lodewijckx C, Sermeus W, et al. Impact of a care pathway for COPD on adherence to guidelines and hospital readmission: a cluster randomized trial. Int J Chron Obstruct Pulmon Dis 2016; 11: 2897-2908.

45 Kruis AL, Smidt N, Assendelft WJ, et al. Integrated disease management interventions for patients with chronic obstructive pulmonary disease. Cochrane Database Syst Rev 2013; 10: CD009437.

46 Jordan RE, Majothi S, Heneghan NR, et al. Supported self-management for patients with moderate to severe chronic obstructive pulmonary disease (COPD): an evidence synthesis and economic analysis. Health Technol Assess 2015; 19: 1-516.

47 Lecheler L, Richter M, Franzen DP, et al. The frequent and under-recognised co-occurrence of acute exacerbated COPD and depression warrants screening: a systematic review. Eur Respir Rev 2017; 26: 170026.

48 Quittner AL, Abbott J, Georgiopoulos AM, et al. International Committee on Mental Health in Cystic Fibrosis: Cystic Fibrosis Foundation and European Cystic Fibrosis Society consensus statements for screening and treating depression and anxiety. Thorax 2016; 71: 26-34.

49 Duff AJ, Bowmer G, Waldron R, et al. Implementing ICMH-CF (International Committee on Mental Health in CF) guidance on screening for depression and anxiety symptoms: a feasibility and pilot study. J Cyst Fibros 2016; 15: e33-e34.

50 Harju E, Michel G, Roser K. A systematic review on the use of the emotion thermometer in individuals diagnosed with cancer. Psychooncology 2019; 28: 1803-1818.

51 Maier C, Aiken L, Busse R. Nurses in Advanced Roles in Primary Care: Policy Levers for Implementation. OECD Health Working Papers Paris, OECD Publishing, 2017. 